\title{
Relationship between dietary sulfur amino acids intake and severity and frequency of pain in patients with musculoskeletal pains
}

niki bahrampour ${ }^{1}$, Ariyo Movahedi ${ }^{2}$, and abolghasem djazayery ${ }^{3}$

${ }^{1}$ Affiliation not available

${ }^{2}$ Islamic Azad University Science and Research Branch

${ }^{3}$ Tehran University of Medical Sciences

April 29, 2021

\begin{abstract}
Background: Musculoskeletal pains (MPs) is a widespread public problem that can affect $13.5 \%$ to $47 \%$ of total population. Dietary changes have strong effects (positive and negative) on a person's health. Sulfur amino acids (SAAs) as a part of protein structure, can be one of the precursors of neurotransmitters, antioxidative metabolic intermediates such as glutathione, effect on inflammation and finally play a role in severity and frequency of MPs. This article will discuss the relationship between dietary sulfur amino acids intake with severity and frequency of pain in patients with MPs. Methods: This study was a matched casecontrol study designed. The target population were 175 men and woman. Anthropometric measurements and pain assessment were collected with questionnaires. Dietary data were collected using 7 days 24-hour recall. ANOVA and Spearman correlation was also performed to examine the relationship between independent and outcome variables. For data analysis, $\mathrm{P}$-value $<0.05$ was considered statistically significant. Results: There was a significant relationship between age, weight, waist circumference (WC), waist circumference to height (WHtR), body mass index (BMI) and severity and frequency of MPs among women. Similarly, in men, there was just a relationship between age and severity of pain. Conclusions: The present study didn't show a positive and relative association between the dietary sulfur amino acids and severity and frequency of pain. What's known? Actually we know SAAs can effect on MPs and inflammation via glutathione synthesis, hyperhomocysteinemia or bone and joint structure. What's new? The relationship between dietary SAAs pattern on inflammation and pain was not statistically significant in this study. More than $98 \%$ of participants consume higher amount of RDA of methionine and cysteine.
\end{abstract}

Relationship between dietary sulfur amino acids intake and severity and frequency of pain in patients with musculoskeletal pains

Niki Bahrampour ${ }^{1}$, Ariyo Movahedi ${ }^{1 *}$, Abolghassem Djazayery ${ }^{2 *}$

${ }^{1}$ Department of Nutrition, Science and Research Branch, Islamic Azad University, Tehran, Iran

${ }^{2}$ Department of Community Nutrition, School of Nutritional Sciences and Dietetics, Tehran University of Medical Sciences

${ }^{*}$ Corresponding authors

Ariyo Movahedi

Email:amm35@mail.aub.edu

Abolghassem Djazayery

Tel: 009821-88955975 
Fax: 009821-88955975

Email: djazayery@yahoo.com

\section{Disclosures relevant to this paper}

The authors declare that they do not have any conflict of interest.

We declare that none of the authors listed on the manuscript are employed by a government agency that has a primary function other than research and/or education. Also we declare that none of the authors are submitting this manuscript as an official representative or on behalf of the government.

Background: Musculoskeletal pains (MPs) is a widespread public problem that can affect $13.5 \%$ to $47 \%$ of total population. Dietary changes have strong effects (positive and negative) on a person's health. Sulfur amino acids (SAAs) as a part of protein structure, can be one of the precursors of neurotransmitters, antioxidative metabolic intermediates such as glutathione, effect on inflammation and finally play a role in severity and frequency of MPs. This article will discuss the relationship between dietary sulfur amino acids intake with severity and frequency of pain in patients with MPs.

Methods: This study was a matched case-control study designed. The target population were 175 men and woman. Anthropometric measurements and pain assessment were collected with questionnaires. Dietary data were collected using 7 days 24-hour recall. ANOVA and Spearman correlation was also performed to examine the relationship between independent and outcome variables. For data analysis, $\mathrm{P}$-value $<0.05$ was considered statistically significant.

Results: There was a significant relationship between age, weight, waist circumference (WC), waist circumference to height (WHtR), body mass index (BMI) and severity and frequency of MPs among women. Similarly, in men, there was just a relationship between age and severity of pain.

Conclusions: The present study didn't show a positive and relative association between the dietary sulfur amino acids and severity and frequency of pain.

Keywords : musculoskeletal pain, sulfur amino acids, diet, pain

\section{What's known? (What is already known about this topic?)}

Although causes of chronic MPs are unknown, but it can be caused by daily life style and diet. In addition many sulfur components are used to have healthier bones and muscles and many sulfur containing supplements are sold to reduce pain in all over the world. Actually we know SAAs can effect on MPs and inflammation via glutathione synthesis, hyperhomocysteinemia or bone and joint structure.

\section{What's new? (What does this article add?)}

Actually the relationship between dietary SAAs intake and severity and frequency of pain is unknown. Consumption too excessive SAAs or lack of intake may can effect on MPs. The relationship between dietary SAAs pattern on inflammation and pain was not statistically significant in this study. More than $98 \%$ of participants consume higher amount of RDA of methionine and cysteine. It means no deficiency of SAAs is found.

\section{Introduction}

Musculoskeletal pain (MPs) is a widespread public problem and there is a need to control effectively and manage severity and frequency of pain in patients. MPs are multifaceted and affect $13.5 \%$ to $47 \%$ of total population(1,2). In the U.S. MPs alone can impose great financial pressure(3). Aging is a strong risk factor for many chronic diseases and pain is the common point between them (4). However, the impact of population aging on the prevalence of pain is not known. MPs especially in lower back, neck, shoulders and knees are major cause of disability throughout life(5-7). Evidences show that most treatments (pharmacological and non- pharmacological intervention) do just a little short-term cure not long-term efficacy for MPs $(7,8)$. Recently, lifestyle changes, poor sleeping, smoking, stress, unhealthy diet and obesity have paid our attention 
to further managing chronic pain(6). Dietary changes have strong effects (positive and negative) on a person's health(9). Among macronutrients, the role of dietary protein can be examined from several aspect like producing the protective layers of bones and joints $(10,11)$ and improving joint/skeletal health(12). Sulfur amino acids (SAAs), as a part of protein structure, (13) can be one of the precursors of neurotransmitters, antioxidative metabolic intermediates such as glutathione and play a role in inflammation, severity and frequency of MPs finally (10). Among SAAs methionine and cysteine are sold as variety of sulfur-containing supplements (Glucosamine sulphate, chondroitin sulfate and methylsulfonylmethane (MSM)) in the markets to reduce chronic inflammatory pain today $(14,15)$. Methionine which is an indispensable amino acid, is resistant to oxidation by reactive oxygen species (ROS) (16) and it is needed for proper growth (17).

It seems that the amount of SAAs received from daily diet is inappropriate with individual needs. So many number of researches report that SAAs consumption have an alleviating action on a large number of chronic diseases and degenerative changes which is associated with normal aging by inhibiting oxidative stress(18). One of the main role of SAAs that is proved in articles is providing sulfates for synthesizing essential metabolic mediators like GAG (glycosaminoglycans), CoA (coenzyme A), SAM (S-Adenosyl-L-Methionine), GSH (glutathione) and etc. in the body. Furthermore Sulfating is necessary for detoxification of many drugs such as acetaminophen which is used widely for relieving of pain by the liver (13). Unfortunately no studies have been performed to address this very important question.

\section{Aim}

This article will discuss the relationship between dietary sulfur amino acids intake with severity and frequency of pain in in patients with MPs.

\section{Methods}

\section{Study population}

This study was a matched case-control study designed and done from February to October 2020. The target population were volunteers referring to physiotherapy and orthopedic clinics in districts 2 and 3 of Tehran, Iran with multistage cluster random sampling. Participants who were enrolled in the study were 175 men and woman above 18 years. Age, sex, education status, occupation, smoking status, menopausal status and delivery number type among women were assessed through a demographic questionnaire.

Having bone fracture in last 3 months, scoliosis, pregnancy and lactation and psychosomatic disorders were the exclusion criteria. Participants were placed into two groups: 85 participants with current musculoskeletal pain as cases and 90 participants with no existence of pain as controls. In order to prevent the effect of confounding factors as much as possible, groups were divided to 18-35, 36-54 and over 55 years through individual matching method.

This study was approved by Ethical Iran National Committee for Ethics in Biomedical Research under code IR.IAU.SRB.REC.1399.084. All the eligible volunteers were informed about the details of the study and in order to sign a written consent their rights were informed to them.

\section{Anthropometric measuring}

Height and weight were measured using Seca 216 to the nearest $0.1 \mathrm{~cm}$ and Seca scale to the nearest 0.1 $\mathrm{kg}$, respectively, while participants stand near the wall with light clothes and no shoes. Waist circumference (WC) was measured in the smallest girth by the expert. Body mass index (BMI) was calculated by dividing weight in kilograms by the square of height in meter.

\section{Pain assessment}

By filling out the McGill Pain Questionnaire through 20 questions, the severity of pain information were received. Scores range were from 0 (no pain) to 78 (severe pain) (19). Qualitative differences in pain might 
be expressed by respondent's questions choice. The frequency of pain was questioned by number of days feeling pain per week. All measurements were done by an expert nurse.

\section{Dietary assessment}

Dietary data were collected using 7days 24 -hour dietary recall through face-to-face interview. They were asked to recall all foods and beverages consumed during the 7 days ago. Portion-sizes of consumed foods were converted to gram. Then each food and beverage was analyzed for their energy and nutrients especially sulfur-containing amino acid (methionine, cysteine) content using Nutritionist IV (version 7.0; N-Squared Computing, Salem, OR). Software program modified for Iranian foods. The software database drawn from United States Department of Agriculture (USDA) food composition tables. Total energy intake between $800-4000 \mathrm{kcal}$ was accepted.

\section{Statistical analysis}

Data analysis was performed using the SPSS version 26 (IBM SPSS Inc.). The Kolmogorov-Smirnov test was used to evaluate the normality of the data. Mean SD (standard deviation) and median (mid-quarter range) were used to describe quantitative variables and frequency report (percentage) for qualitative variables between case and control groups. Independent t-test was used to compare the mean of quantitative outcomes between the two groups. Chi-square test was used to compare qualitative factors between the two groups. ANCOVA test was used to adjust confounders such as age, body mass index (BMI) and energy intake. The comparison of the mean of quantitative variables between the tertiles of SAAs intakes was evaluated by ANOVA with GLM tests.

Spearman correlation was also performed to examine the relationship between independent and outcome factors. For data analysis, the P-value $<0.05$ was considered statistically significant.

\section{Results}

\section{Study population and general characteristics}

General characteristics among participants were shown in Table 1. The range of age in this study population was above 18 years and $39 \%$ of members were above 35 years. Only $28.6 \%$ of participants were male. The mean \pm standard deviation (SD) of BMI and daily energy intake were $24.84 \pm 4.32 \mathrm{~kg} / \mathrm{m}^{2}$ and $2230 \pm 651.71$ $\mathrm{kcal} /$ day respectively. No severe pain was detected in adults.

Table1 characteristics of study participants among of total SAAs intake tertiles

\begin{tabular}{|c|c|c|c|c|c|c|}
\hline Variables & Variables & Variables & $\begin{array}{l}\text { SAAs } \\
\text { intake } \\
\text { Q1 }(n=58)\end{array}$ & $\begin{array}{l}\text { SAAs } \\
\text { intake } \\
\text { Q2 }(n=59)\end{array}$ & $\begin{array}{l}\text { SAAs } \\
\text { intake } \\
\text { Q3 }(n=58)\end{array}$ & $\begin{array}{l}\text { SAAs } \\
\text { intake } \\
\mathbf{P}^{*} \text { value }\end{array}$ \\
\hline Total & Total & Total & Total & Total & Total & $<0.001$ \\
\hline $\begin{array}{l}\text { SAAs } \\
(\mathrm{mg} / \text { day })\end{array}$ & $\begin{array}{l}\text { SAAs } \\
(\mathrm{mg} / \text { day })\end{array}$ & $\begin{array}{l}\text { SAAs } \\
(\mathbf{m g} / \text { day }) \\
\text { Median }\end{array}$ & $\begin{array}{l}\text { SAAs } \\
(\mathbf{m g} / \mathbf{d a y}) \\
1981.29^{\mathrm{a}}\end{array}$ & $\begin{array}{l}\text { SAAs } \\
(\mathbf{m g} / \mathbf{d a y}) \\
2968.39^{\mathrm{b}}\end{array}$ & $\begin{array}{l}\text { SAAs } \\
(\mathbf{m g} / \mathbf{d a y}) \\
4646.96^{\mathrm{c}}\end{array}$ & \\
\hline $\begin{array}{l}\text { Gender } \\
\mathbf{n}(\%)\end{array}$ & $\begin{array}{l}\text { Gender } \\
\mathbf{n}(\%)\end{array}$ & $\begin{array}{l}\text { Gender } \\
\mathbf{n}(\%) \\
\text { Men } \\
\text { Woman }\end{array}$ & $\begin{array}{l}\text { Gender } \\
\mathbf{n}(\%) \\
5(8.5)^{\mathrm{a}} \\
54(91.5)^{\mathrm{a}}\end{array}$ & $\begin{array}{l}\text { Gender } \\
\mathbf{n}(\%) \\
17(29.3) \mathrm{b} \\
41(70.7)^{\mathrm{b}}\end{array}$ & $\begin{array}{l}\text { Gender } \\
\mathbf{n}(\%) \\
28(48.3)^{c} \\
30(51.7)^{c}\end{array}$ & $<0.001$ \\
\hline $\begin{array}{l}\text { Smoking } \\
\mathbf{n}(\%)\end{array}$ & $\begin{array}{l}\text { Smoking } \\
\mathbf{n}(\%)\end{array}$ & $\begin{array}{l}\text { Smoking } \\
\mathbf{n}(\%) \\
\text { Yes } \\
\text { No }\end{array}$ & $\begin{array}{l}\text { Smoking } \\
\mathbf{n}(\%) \\
5(8.5) \\
54(91.5)\end{array}$ & $\begin{array}{l}\text { Smoking } \\
\text { n(\%) } \\
12(20.7) \\
40(69.0)\end{array}$ & $\begin{array}{l}\text { Smoking } \\
\mathbf{n}(\%) \\
12(15.5) \\
47(81.0)\end{array}$ & 0.65 \\
\hline
\end{tabular}




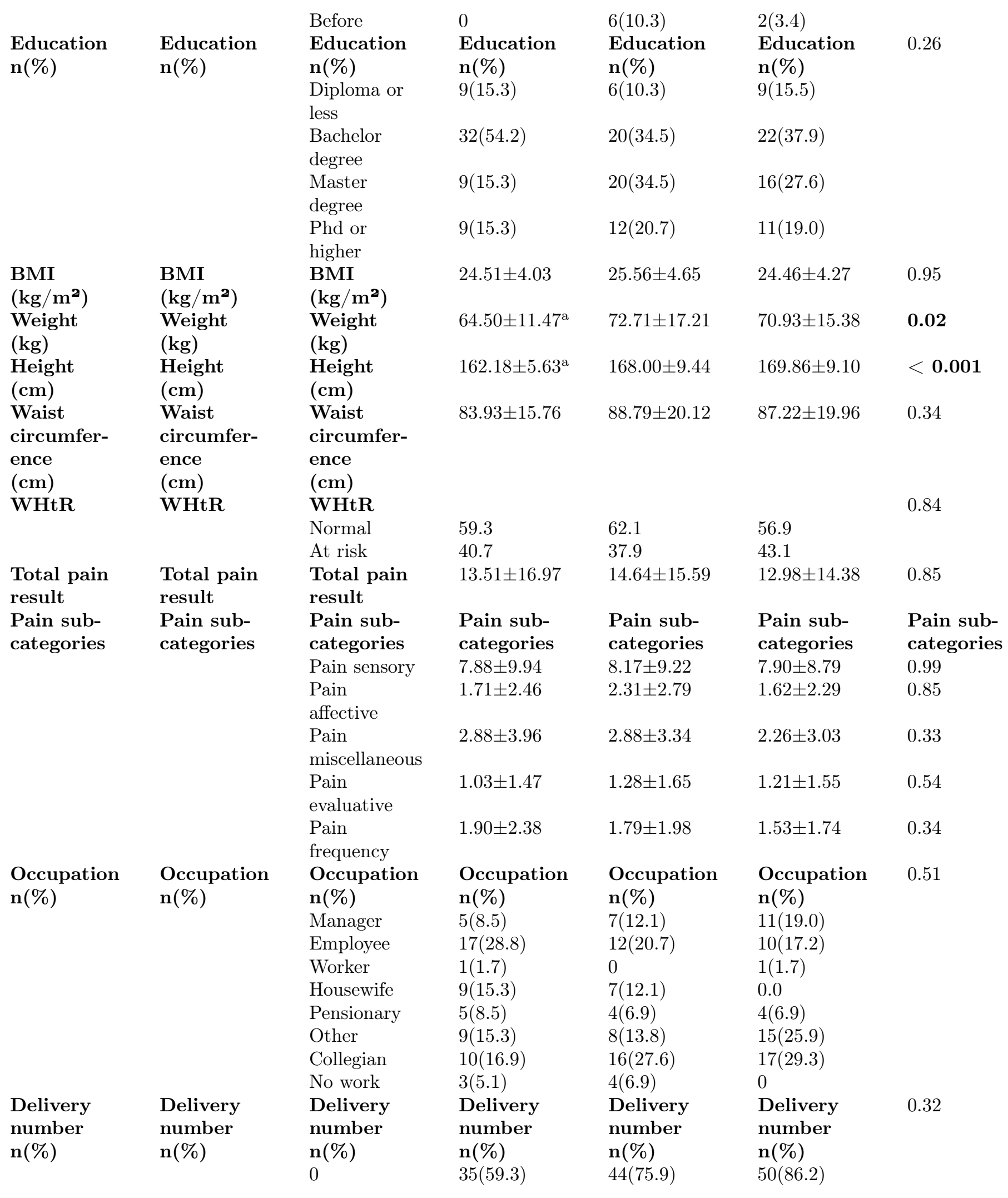




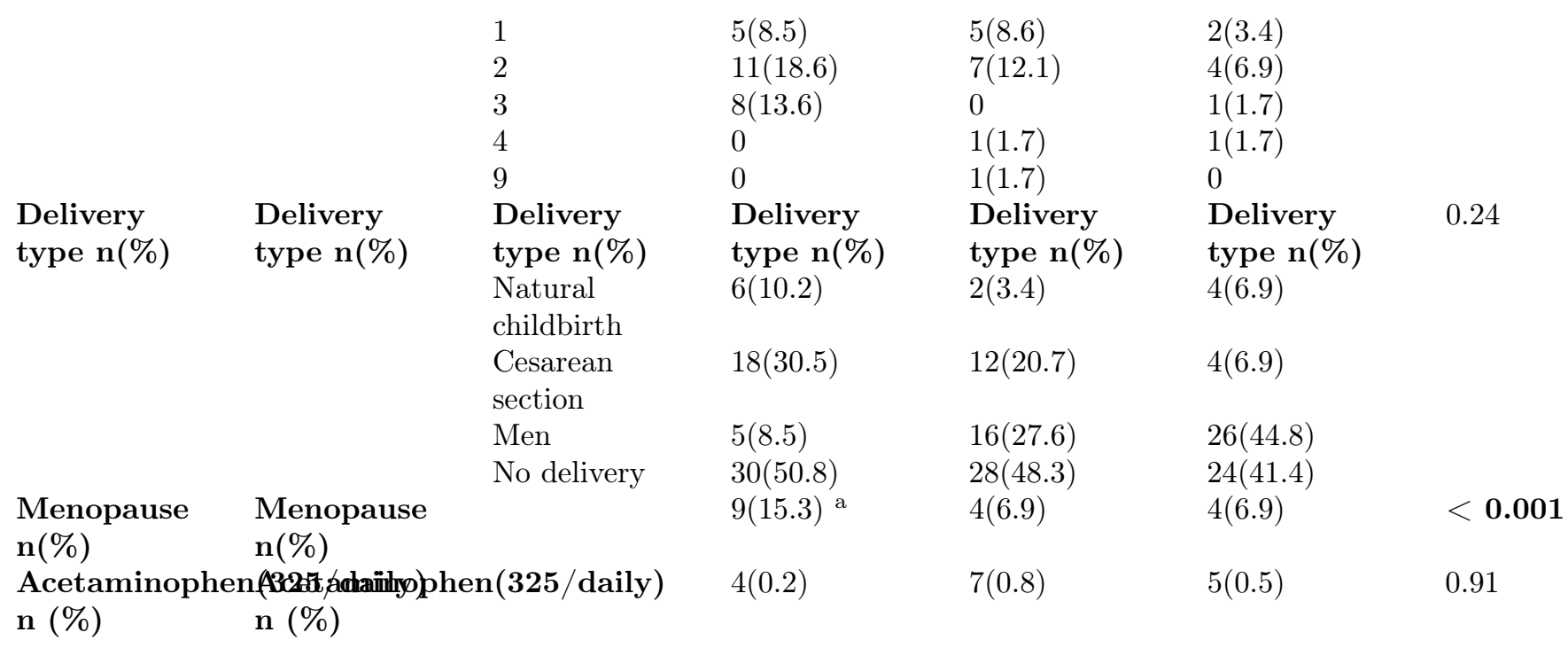




\begin{tabular}{|c|c|c|c|c|c|c|}
\hline & & & & & & \\
\hline & & & & & & \\
\hline 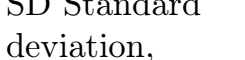 & $\begin{array}{l}\text { SD Standard } \\
\text { deviation, }\end{array}$ & $\begin{array}{l}\text { SD Stan } \\
\text { deviatio }\end{array}$ & dard & & tard & ard \\
\hline BMI: body & BMI: body & BMI: body & & & & \\
\hline & & & & & & \\
\hline WHtR: waist & WHtR: waist & WHtR: waist & WHtR: waist & WHtR: waist & WHtR: waist & WHtR: waist \\
\hline $\begin{array}{l}\text { to helght ratio, } \\
\text { Quantitative }\end{array}$ & $\begin{array}{l}\text { to height ratio, } \\
\text { Quantitative }\end{array}$ & $\begin{array}{l}\text { to height ratio, } \\
\text { Quantitative }\end{array}$ & $\begin{array}{l}\text { to helght ratio, } \\
\text { Quantitative }\end{array}$ & $\begin{array}{l}\text { to height ratio, } \\
\text { Quantitative }\end{array}$ & $\begin{array}{l}\text { to height ratio, } \\
\text { Quantitative }\end{array}$ & $\begin{array}{l}\text { to helght ratio, } \\
\text { Quantitative }\end{array}$ \\
\hline variables were & variables were & variables were & variables were & variables were & variables were & variables were \\
\hline $\begin{array}{l}\text { showed by } \\
\text { means } \pm \text { SD }\end{array}$ & $\begin{array}{l}\text { snowed by } \\
\text { means } \pm \text { SD }\end{array}$ & $\begin{array}{l}\text { snowed by } \\
\text { means } \pm \text { SD }\end{array}$ & $\begin{array}{l}\text { snowed by } \\
\text { means } \pm \text { SD }\end{array}$ & $\begin{array}{l}\text { snowed by } \\
\text { means } \pm \text { SD }\end{array}$ & $\begin{array}{l}\text { snowed by } \\
\text { means } \pm \text { SD }\end{array}$ & $\begin{array}{l}\text { snowed by } \\
\text { means } \pm \text { SD }\end{array}$ \\
\hline and qualitative & and qualitative & and qualitative & and qualitative & and qualitative & and qualitative & and qualitative \\
\hline $\begin{array}{l}\text { variables were } \\
\text { showed by }\end{array}$ & $\begin{array}{l}\text { variables were } \\
\text { showed by }\end{array}$ & & & & $\begin{array}{l}\text { variables were } \\
\text { showed by }\end{array}$ & were \\
\hline number & r & & & & & \\
\hline (percentage). & (perc & (per & (p & age). & $(\mathrm{p}$ & age). \\
\hline $\mathrm{P}$ value $<0.05$ & $\mathrm{P}$ value $<0.05$ & $<0.05$ & $<0.05$ & $\mathrm{P}$ val & .05 & $e<0.05$ \\
\hline & & & & & & \\
\hline significant. ${ }^{*} \mathrm{P}$ & significant. ${ }^{*} \mathrm{P}$ & significant. ${ }^{*} \mathrm{P}$ & significant. ${ }^{*} \mathrm{P}$ & significant. ${ }^{*} \mathrm{P}$ & significant. ${ }^{*} \mathrm{P}$ & significant. ${ }^{*} \mathrm{P}$ \\
\hline $\begin{array}{l}\text { values resulted } \\
\text { from }\end{array}$ & $\begin{array}{l}\text { values resulted } \\
\text { from }\end{array}$ & $\begin{array}{l}\text { values resulted } \\
\text { from }\end{array}$ & $\begin{array}{l}\text { values resulted } \\
\text { from }\end{array}$ & es resulted & $\begin{array}{l}\text { values resulted } \\
\text { from }\end{array}$ & $\begin{array}{l}\text { lues resulted } \\
\text { om }\end{array}$ \\
\hline ANCOVA & ANCOVA & ANCOVA & ANCOVA & ANCOVA & ANCOVA & ANCOVA \\
\hline analysis and & ana & ar & a & and & $\mathrm{a}$ & nd \\
\hline were adjusted & usted & usted & & usted & usted & justed \\
\hline for energy & & & & & & \\
\hline $\begin{array}{l}\text { intake. } \\
\text { abc Different }\end{array}$ & $\begin{array}{l}\text { inta } \\
a b c\end{array}$ & & & & & \\
\hline etters in each & in each & letters in each & letters in each & tters in each & $n$ each & in each \\
\hline & & & & & & \\
\hline fference & ifference & ference & Ference & ference & fference & ifference \\
\hline between & between & between & between & between & between & between \\
\hline groups based & groups based & groups based & groups based & groups based & oups based & oups based \\
\hline & & & & & $\mathrm{O}$ & on $\mathrm{A}$ \\
\hline & & & & & & \\
\hline Duncan post & Duncan post & Duncan post & Duncan post & Duncan post & Duncan post & Duncan post \\
\hline hoc tes & & & & & ct $(\mathrm{n}$ & est (p \\
\hline$<0.05)$ & $<0.05)$ & $<0.05)$ & $<0.05)$ & & $<0.00)$ & \\
\hline
\end{tabular}

\section{Comparison of daily nutrient intake in participants across people in pain tertiles}

In table 2, the intakes of food groups and macro and micro nutrients are shown after adjusting for sex, BMI and energy among pain tertils. Firs tertile group includes healthy people with no feeling of pain. Range of SAAa intake was between 652.72 to $17841 \mathrm{~g}$ /day. A positive relation had found between energy, carbohydrate and $\mathrm{B} 1$ intake among three groups $(\mathrm{P}<0.05)$.

Table2 comparison of daily nutrient intake in participants across people in pain tertiles 


\begin{tabular}{|c|c|c|c|c|c|c|}
\hline \multirow{2}{*}{$\begin{array}{l}\text { Variables } \\
\text { Amounts per } \\
\text { day }\end{array}$} & \multirow[b]{2}{*}{$\begin{array}{l}\text { Q1 } \\
(n=90)\end{array}$} & \multicolumn{2}{|l|}{ Q2 $(n=55)$} & \multirow[b]{2}{*}{$\mathbf{P}$ trend } & \multirow[b]{2}{*}{$\mathbf{P}$ value $^{\mathrm{a}}$} & \multirow[b]{2}{*}{$P$ value $^{a}$} \\
\hline & & & $\begin{array}{l}\text { Q3 } \\
(\mathrm{n}=30)\end{array}$ & & & \\
\hline & $2147.64 \pm 670.41$ & $2388.54 \pm 629.30$ & $2191.66 \pm 601.17$ & 0.03 & 0.03 & 0.09 \\
\hline \multicolumn{7}{|l|}{$\begin{array}{l}\text { Energy } \\
\text { (kcal) }\end{array}$} \\
\hline Protein (g) & $93.76 \pm 57.44$ & $106.85 \pm 87.66$ & $88.29 \pm 31.64$ & 0.24 & 0.24 & 0.36 \\
\hline $\begin{array}{l}\text { Carbohydrate } \\
(\mathrm{g})\end{array}$ & $229.45 \pm 94.32$ & $266.99 \pm 108.42$ & $253.79 \pm 102.37$ & 0.03 & 0.03 & 0.09 \\
\hline Fat $(\mathrm{g})$ & $102.40 \pm 44.93$ & $108.10 \pm 42.31$ & $100.52 \pm 24.23$ & 0.42 & 0.42 & 0.57 \\
\hline $\begin{array}{l}\text { Cholesterol } \\
(\mathrm{mg})\end{array}$ & $345.89 \pm 253.87$ & $350.19 \pm 280.76$ & $337.19 \pm 269.04$ & 0.92 & 0.92 & 0.97 \\
\hline SFA (g) & $29.99 \pm 23.46$ & $27.27 \pm 9.56$ & $29.07 \pm 14.23$ & 0.39 & 0.39 & 0.57 \\
\hline PUFA (g) & $25.90 \pm 13.82$ & $30.71 \pm 23.26$ & $25.89 \pm 7.28$ & 0.09 & 0.09 & 0.34 \\
\hline $\begin{array}{l}\text { alpha- } \\
\text { Linolenic } \\
\text { acid (mg) }\end{array}$ & $3.21 \pm 8.98$ & $3.30 \pm 9.35$ & $4.45 \pm 9.47$ & 0.95 & 0.95 & 0.81 \\
\hline $\begin{array}{l}\text { Sodium } \\
(\mathrm{mg})\end{array}$ & $1790.44 \pm 831.05$ & $2127.07 \pm 1194.83$ & $1859.41 \pm 825.34$ & 0.04 & 0.04 & 0.19 \\
\hline $\begin{array}{l}\text { Potassium } \\
(\mathrm{mg})\end{array}$ & $3670.49 \pm 1485.21$ & $3912.67 \pm 1530.67$ & $3966.11 \pm 1702.20$ & 0.35 & 0.35 & 0.53 \\
\hline $\begin{array}{l}\text { Calcium } \\
(\mathrm{mg})\end{array}$ & $14.34 \pm 6.26$ & $16.42 \pm 6.94$ & $16.29 \pm 8.16$ & 0.07 & 0.07 & 0.15 \\
\hline $\begin{array}{l}\text { Magnesium } \\
(\mathrm{mg})\end{array}$ & $1226.86 \pm 596.75$ & $1251.81 \pm 490.32$ & $1158.05 \pm 562.11$ & 0.79 & 0.79 & 0.74 \\
\hline $\begin{array}{l}\text { Phosphorus } \\
(\mathrm{mg})\end{array}$ & $1530.49 \pm 680.62$ & $1637.88 \pm 742.92$ & $1518.96 \pm 554.84$ & 0.35 & 0.35 & 0.63 \\
\hline $\begin{array}{l}\text { VitaminA } \\
\text { (RAE) }\end{array}$ & $2143.24 \pm 1378.57$ & $2194.53 \pm 1583.94$ & $2187.81 \pm 1129.64$ & 0.83 & 0.83 & 0.97 \\
\hline B1 (mg) & $1.61 \pm 0.64$ & $1.87 \pm 0.80$ & $1.70 \pm 0.58$ & 0.02 & 0.02 & 0.11 \\
\hline B2 (mg) & $2.49 \pm 1.03$ & $2.65 \pm 0.96$ & $2.41 \pm 0.89$ & 0.32 & 0.32 & 0.46 \\
\hline B3 (mg) & $22.48 \pm 15.39$ & $26.16 \pm 21.88$ & $21.50 \pm 7.56$ & 0.20 & 0.20 & 0.36 \\
\hline B6 (mg) & $2.15 \pm 1.11$ & $2.44 \pm 1.62$ & $2.31 \pm 1.39$ & 0.21 & 0.21 & 0.49 \\
\hline B9 $(\mu g)$ & $341.64 \pm 179.25$ & $343.23 \pm 142.97$ & $405.87 \pm 316.22$ & 0.96 & 0.96 & 0.56 \\
\hline B12 ( $\mu \mathrm{g})$ & $7.01 \pm 6.56$ & $6.49 \pm 5.27$ & $5.82 \pm 4.94$ & 0.60 & 0.60 & 0.58 \\
\hline $\begin{array}{l}\text { VitaminD } \\
(\mu \mathrm{g})\end{array}$ & $2.69 \pm 2.04$ & $2.66 \pm 2.30$ & $2.34 \pm 1.99$ & 0.91 & 0.91 & 0.69 \\
\hline Fiber (g) & $13.99 \pm 9.62$ & $15.53 \pm 9.03$ & $17.75 \pm 15.59$ & 0.40 & 0.40 & 0.36 \\
\hline Fructose (g) & $7.18 \pm 6.12$ & $7.88 \pm 5.51$ & $8.79 \pm 9.80$ & 0.53 & 0.53 & 0.60 \\
\hline Sucrose (g) & $15.44 \pm 10.88$ & $15.49 \pm 8.06$ & $16.47 \pm 11.99$ & 0.97 & 0.97 & 0.91 \\
\hline Lactose (g) & $19.05 \pm 12.37$ & $18.61 \pm 12.01$ & $15.64 \pm 11.11$ & 0.82 & 0.82 & 0.36 \\
\hline $\begin{array}{l}\text { Tryptophan } \\
\text { (mg) }\end{array}$ & $1023.78 \pm 654.18$ & $1171.85 \pm 1010.08$ & $970.15 \pm 347.16$ & 0.25 & 0.25 & 0.40 \\
\hline $\begin{array}{l}\text { Caffeine } \\
(\mathrm{mg})\end{array}$ & $185.28 \pm 132.90$ & $175.90 \pm 123.48$ & $226.54 \pm 134.59$ & 0.67 & 0.67 & 0.23 \\
\hline $\begin{array}{l}\text { Methionine } \\
(\mathrm{mg})\end{array}$ & $2164.71 \pm 1557.72$ & $2269.54 \pm 1479.06$ & $1920.50 \pm 773.55$ & 0.66 & 0.66 & 0.30 \\
\hline
\end{tabular}




\begin{tabular}{|c|c|c|c|c|c|c|}
\hline $\begin{array}{l}\text { Cysteine } \\
(\mathrm{mg})\end{array}$ & $1176.10 \pm 717.85$ & $1285.38 \pm 748.37$ & $1128.08 \pm 417.79$ & 0.35 & 0.35 & 0.46 \\
\hline \multirow{2}{*}{$\begin{array}{l}\text { Abbreviations } \\
\text { SD: standard } \\
\text { deviation, }\end{array}$} & \multirow{2}{*}{$\begin{array}{l}\text { Abbreviations: } \\
\text { SD: standard } \\
\text { deviation, }\end{array}$} & \multirow{2}{*}{$\begin{array}{l}\text { Abbreviations: } \\
\text { SD: standard } \\
\text { deviation, }\end{array}$} & \multirow{2}{*}{$\begin{array}{l}\text { Abbreviations: } \\
\text { SD: standard } \\
\text { deviation, }\end{array}$} & \multirow{2}{*}{$\begin{array}{l}\text { Abbreviations } \\
\text { SD: standard } \\
\text { deviation, }\end{array}$} & \multirow{2}{*}{$\begin{array}{l}\text { Abbreviations: } \\
\text { SD: standard } \\
\text { deviation, }\end{array}$} & \multirow{2}{*}{$\begin{array}{l}\text { Abbreviations } \\
\text { SD: standard } \\
\text { deviation, }\end{array}$} \\
\hline & & & & & & \\
\hline $\begin{array}{l}\text { SFA: Satu- } \\
\text { rated fat, } \\
\text { PUFA: } \\
\text { Polyun- } \\
\text { saturated } \\
\text { fatty acids, } \\
\text { Data are } \\
\text { presented as }\end{array}$ & $\begin{array}{l}\text { SFA: Satu- } \\
\text { rated fat, } \\
\text { PUFA: } \\
\text { Polyun- } \\
\text { saturated } \\
\text { fatty acids, } \\
\text { Data are } \\
\text { presented as }\end{array}$ & $\begin{array}{l}\text { SFA: Satu- } \\
\text { rated fat, } \\
\text { PUFA: } \\
\text { Polyun- } \\
\text { saturated } \\
\text { fatty acids, } \\
\text { Data are } \\
\text { presented as }\end{array}$ & $\begin{array}{l}\text { SFA: Satu- } \\
\text { rated fat, } \\
\text { PUFA: } \\
\text { Polyun- } \\
\text { saturated } \\
\text { fatty acids, } \\
\text { Data are } \\
\text { presented as }\end{array}$ & $\begin{array}{l}\text { SFA: Satu- } \\
\text { rated fat, } \\
\text { PUFA: } \\
\text { Polyun- } \\
\text { saturated } \\
\text { fatty acids, } \\
\text { Data are } \\
\text { presented as }\end{array}$ & $\begin{array}{l}\text { SFA: Satu- } \\
\text { rated fat, } \\
\text { PUFA: } \\
\text { Polyun- } \\
\text { saturated } \\
\text { fatty acids, } \\
\text { Data are } \\
\text { presented as }\end{array}$ & $\begin{array}{l}\text { SFA: Satu- } \\
\text { rated fat, } \\
\text { PUFA: } \\
\text { Polyun- } \\
\text { saturated } \\
\text { fatty acids, } \\
\text { Data are } \\
\text { presented as }\end{array}$ \\
\hline $\begin{array}{l}\text { Mean } \pm \text { SD. P } \\
\text { values resulted } \\
\text { from ANOVA } \\
\text { analysis. }\end{array}$ & $\begin{array}{l}\text { Mean } \pm \text { SD. P } \\
\text { values resulted } \\
\text { from ANOVA } \\
\text { analysis. }\end{array}$ & $\begin{array}{l}\text { Mean } \pm \text { SD. P } \\
\text { values resulted } \\
\text { from ANOVA } \\
\text { analysis. }\end{array}$ & $\begin{array}{l}\text { Mean } \pm \text { SD. P } \\
\text { values resulted } \\
\text { from ANOVA } \\
\text { analysis. }\end{array}$ & $\begin{array}{l}\text { Mean } \pm \text { SD. P } \\
\text { values resulted } \\
\text { from ANOVA } \\
\text { analysis. }\end{array}$ & $\begin{array}{l}\text { SD. P } \\
\text { esulted } \\
\text { NOVA }\end{array}$ & $\begin{array}{l}\text { SD. P } \\
\text { esulted } \\
\text { NOVA } \\
\end{array}$ \\
\hline $\begin{array}{l}\mathrm{P} \text {-value }<0.05 \\
\text { is significant. } \\
{ }^{*} \mathrm{P} \text { values } \\
\text { presented } \\
\text { resulted from }\end{array}$ & $\begin{array}{l}\mathrm{P} \text {-value }<0.05 \\
\text { is significant. } \\
* \mathrm{P} \text { values } \\
\text { presented } \\
\text { resulted from }\end{array}$ & $\begin{array}{l}\mathrm{P} \text {-value }<0.05 \\
\text { is significant. } \\
{ }^{*} \mathrm{P} \text { values } \\
\text { presented } \\
\text { resulted from }\end{array}$ & $\begin{array}{l}\mathrm{P} \text {-value }<0.05 \\
\text { is significant. } \\
{ }^{*} \mathrm{P} \text { values } \\
\text { presented } \\
\text { resulted from }\end{array}$ & $\begin{array}{l}\mathrm{P} \text {-value }<0.05 \\
\text { is significant. } \\
* \mathrm{P} \text { values } \\
\text { presented } \\
\text { resulted from }\end{array}$ & $\begin{array}{l}e<0.05 \\
\text { ificant. } \\
\text { ues } \\
\text { ted } \\
\text { d from }\end{array}$ & $\begin{array}{l}\text { value }<0.05 \\
\text { significant. } \\
\text { values } \\
\text { esented } \\
\text { sulted from }\end{array}$ \\
\hline $\begin{array}{l}\text { ANCOVA } \\
\text { analysis. }\end{array}$ & $\begin{array}{l}\text { ANCOVA } \\
\text { analysis. }^{\text {a }}\end{array}$ & $\begin{array}{l}\text { ANCOVA } \\
\text { analysis. }^{\text {a }}\end{array}$ & $\begin{array}{l}\text { ANCOVA } \\
\text { analysis. }^{\text {a }}\end{array}$ & $\begin{array}{l}\text { ANCOVA } \\
\text { analysis. }^{\text {a }}\end{array}$ & $\begin{array}{l}\text { ANCOVA } \\
\text { analysis. }^{\text {a }}\end{array}$ & $\begin{array}{l}\text { COVA } \\
\text { alysis. a }\end{array}$ \\
\hline & & & & & & \\
\hline $\begin{array}{l}\text { equalizing the } \\
\text { variables based }\end{array}$ & & & & & & \\
\hline $\begin{array}{l}\text { sex, } \\
\text { lass }\end{array}$ & $\begin{array}{l}\text { on age, sex, } \\
\text { body mass }\end{array}$ & e, sex & $\begin{array}{l}\text { on age, sex, } \\
\text { body mass }\end{array}$ & $\begin{array}{l}\text { on age, sex, } \\
\text { body mass }\end{array}$ & $\begin{array}{l}\text { ge, sex, } \\
\text { y mass }\end{array}$ & $\begin{array}{l}\mathrm{x} \\
\mathrm{s}\end{array}$ \\
\hline ased on & index based on & index based on & index based on & index based on & index based on & index based on \\
\hline $\begin{array}{l}\text { were evaluated } \\
\text { using GLM }\end{array}$ & $\begin{array}{l}\text { were evaluated } \\
\text { using GLM }\end{array}$ & $\begin{array}{l}\text { were evaluated } \\
\text { using GLM }\end{array}$ & $\begin{array}{l}\text { were evaluated } \\
\text { using GLM }\end{array}$ & $\begin{array}{l}\text { were evaluated } \\
\text { using GLM }\end{array}$ & $\begin{array}{l}\text { were evaluated } \\
\text { using GLM }\end{array}$ & $\begin{array}{l}\text { were evaluated } \\
\text { using GLM }\end{array}$ \\
\hline
\end{tabular}

Correlation between pain frequency and the studied variables by divided gender

To investigate the relationship between the amount of sulfur amino acids and pain, spearman correlation was applied in Table 3 and 4. After adjusting for potential confounding variable included total energy intake, there was a significant relationship and weak correlation between age, weight, WC, WHtR and BMI and severity and frequency of MPs among women. Similarly, in men, there was just a positive and strong relationship between age and severity of pain $(\mathrm{R}=0.36 ; \mathrm{P}=0.01)$. No positive relationship between any of the anthropometric indicators and strength and frequency of pain was seen. In addition there was no relationship between daily SAAs intake and severity of pain totally $(\mathrm{P}>0.05)$. Finally, the relationship between age, WC, WHtR and BMI with pain intensity and age, WHtR, BMI and pain frequency was statistically significant.

Table 3 Correlation between pain frequency and the studied variables by divided gender

Variables $^{a}$

SAAs intake 


\section{Weight \\ Height \\ WC \\ WHtR \\ BMI}

Age

Abbreviations: SAAs: sulfur amino acids, WHtR: waist to height, BMI: body mass index, WC: waist circumference * Signi

Table 4 Correlation between pain intensity and the studied variables by divided gender

variable $^{a}$

Total SAAs intake

Weight

Height

WC

WHtR

BMI

Age

Abbreviations: SAAs: sulfur amino acids, WHtR: waist to height, BMI: body mass index, WC: waist circumference * Signi

\section{Discussion}

The current study explored the relationship of dietary sulfur amino acids intake and severity and frequency of MPs in adults. According to the results, there was not any significant relationship between sulfur amino acids consumption and severity and frequency of MPs; whereas higher intake of protein was related to the higher feeling of pain interceded by calorie intake. In addition, almost $71 \%$ of adults felt low to moderate pain. In the present study, there was no significant relationship between gender and the severity of musculoskeletal pain in the adult population over 18 years. Neither Palmer and Goodson did not see any differences in review article, nor did Roberts et al $(20,21)$. But there was a positive relation in frequency of pain among women so that $56 \%$ of women felt pain more than twice a week. It has been considered that about $60 \%$ of the participants were young and age has a significant positive relationship with the severity and frequency of pain in individuals (22). Increasing pain with getting older can effect on progressive loss of neuromuscular function, decreased muscle mass, cartilage, bone mass and skeletal and muscular degenerative injuries (23). Recently, more attention has been paid to the effect of sex hormones on pain induction. Microglia (the remnant of the components of the immune system in the central nervous system) play a vital role in inducing pain in the body differently in men and women (24). As we know, in the central hypothalamus Arcuate Nucleus produce beta-endorphin, a potent analgesic for relieving of chronic pain in the body, is strongly influenced by estrogen. Estrogen receptors influence in synthesis and secretion of the methionine-enkephalin (delta-epithelial ligand receptor) and beta-endorphin secretion. This is why women feel less pain during pregnancy (25).

In the present study, there was a strong correlation between weight, waist circumference, waist height and body mass index with the severity and frequency of musculoskeletal pain in women. Also, in men, there was no significant relationship between any of the anthropometric indicators with pain intensity and frequency. It should be noted that the number of female participants in this study was higher and this could be one of the possible reasons for existence of significant pain with anthropometric figures in women. Also, the percentage of body fat mass is higher in women, which can be a factor in shortening the height between discs and increasing the severity of pain among them (26). Weight loss and healthy eating pattern may be helpful to manage obesity, reduce inflammation and disability of pain (27). In addition, endogenous enkephalins 
have been shown to increase food intake in cats, thereby increasing obesity and pain (28). One study have also shown that the expression of catecholamine-stimulating lipolytic enzymes and the hormone-sensitive lipase was inhibited in adipose tissue and total body weight and three glyceride level were increased in the liver of mice with a high cysteine diet after 12-weeks. In general, excessive consumption of cysteine causes obesity and undesirable metabolic phenotype in mice. Cysteine plasma and obesity in humans may be causal (29).

There was mostly high sulfur amino acids among participants even more than RDA determinate (14 $\mathrm{mg} / \mathrm{kg} /$ day) (30). In line with this study, in a systematic review Elma et al. found that in most patients with pain, the protein intake was higher than the allowable intake but the severity of pain was not significantly related to dietary intake (9). Although protein is a major component of maintaining skeletal muscle health, most sulfur-rich sources of amino acids are found in animal proteins, especially red meat and poultry which can be lead to inflammation (31). In addition, the consumption of proteins, especially from animal origin, more than 0.8 grams per kilogram of body weight has no positive effects on human health. Animal proteins in the body produce acidic precursors due to incomplete oxidation and plant proteins produce alkaline precursors in the body. An imbalance between acidic and alkaline precursors has been shown to disrupt a chronic net dietary acid load, which may have adverse consequences on bone health (32). Also Hackney et al. found that excessive consumption of sulfur amino acids was associated with increased bone resorption. Dietary methionine can decrease blood $\mathrm{pH}$ and increases skeletal pain. Consistent with the present study in the study of Freyberg et al. which was conducted to find the relationship between dietary sulfur amino acids and rheumatoid arthritis, no sulfur amino acid deficiency was observed in people with arthritis (33). In a cohort study with 546 people with rheumatoid arthritis, no significant correlation was observed between the type of protein consumed and the risk of rheumatoid arthritis $(34,35)$. There was just a positive relationship between carbohydrate intake and the group that feel most pain.

In addition the internal pain relief system in central nerves system need essential fatty acids, especially Eicosapentaenoic acid (EPA) and Docosahexaenoic acid (DHA) (36,37). Whereas the consumption of omega3 fats (high in fatty fish) was very low in the population of this study. In addition consumption of salt was positively correlated with pain.

These two are precursors of S-adenosyl methionine (SAM), hydrogen sulfide, taurine and glutathione which can are protective $(38,39)$. Oral cysteine alone has been shown to have low absorption capacity from the gastrointestinal tract $(40)$.

In addition taking high dose of oral methionine may increase the level of homocysteine and could be harmful for persons. Bioavailability of cysteine is a limiting step in glutathione production. One study showed that oral cysteine alone has been absorbed in a low capacity from the gastrointestinal tract. In order to increase the level of glutathione, whey protein and almond could be useful (40).

Homocysteine is one of the mediators of methionine metabolism in the body. This substance has many roles in transporting in cells. In muscle disorders, for examples, the role of homocysteine as an aggravator of key processes such as oxidative stress, nitric oxide (NO) signaling, endoplasmic reticulum stress, methylation and gene expression, alteration in cellular signaling, especially G proteins, is prominent (41).

Among painkillers drugs no relationship between was seen acetaminophen and SAAa. Glutathione detoxify drugs such as acetaminophen by the sulfur element $(42,43)$. In line with this study, an intervention study was conducted in 2012. They selected 11 people over the age of 69 who had arthritis. Participants received 3 grams of acetaminophen per day for 14 days. They found that despite the excretion of small amounts of paracetamol as conjugated sulfate in elderly patients, the amount of sulfur required to detoxify $3 \mathrm{~g}$ of paracetamol per day was equal to $20 \%$ of the sulfur supplied by $1.06 \mathrm{~g} / \mathrm{kg}$ body weight of dietary protein. Also, after 14 days, the body's total protein stores remained unchanged. However, there was an increase in the oxidation of sulfur amino acids and a decrease in the body's antioxidant capacity. The safety/risk ratio of this drug to compensate for deficiencies may be greatly need via new dietary changes and guidelines (44). Hyperhomocysteinemia can be a risk factor for pathology of many diseases. Homocysteine can damage 
the vagus nerve and the superior cervical ganglion in the central nervous system and disrupt both the sympathetic and parasympathetic systems involved in skeletal muscle, which can lead to dysfunction of the skeletal muscle and spinal cord in the long term (45). In addition, the body's autonomic system and adrenal glands need methionine to produce norepinephrine from epinephrine in process of methylation (46). In chronic pain, norepinephrine increases inflammation in the body by producing pro-inflammatory cytokines. Due to the fact that the consumption of sulfur amino acids in this study was high, norepinephrine may have increased chronic pain by producing more inflammatory cytokines (47).

Conclusion

In conclusion, the present study didn't show a positive and relative association between the dietary sulfur amino acids and severity and frequency of pain. Although, targeting a decrease in dietary animal protein intake might be a useful strategy for reducing pain. However, further studies are recommended to improve understanding the relationship and managing pain.

Strengths and Limitations: According to the searched data bank, this study was the one which had focused the role of dietary SAAs and existence of pain in individuals. However, the population of this study was mostly among adults group (18-35 years) and assessing to older people in quarantine period of covid-19 was difficult for us and this may influence the final result. Further research is recommended to concrete our result.

\section{Ethics approval and consent to participate}

The National Committee for Ethics in Biomedical Research approved this study under code IR.IAU.SRB.REC.1399.084. The specifics of the study were told to all qualified participants and written consent was obtained. The data are not publicly available because of containing information that could compromise the privacy of the research. Data are available from the authors upon reasonable request and with the permission of Ariyo Movahedi.

\section{Availability of data and materials}

Data supporting the results of this study are available from the Islamic Azad University of Science and Research Branch (SRBIAU) and have been used under license for the current analysis. However, data are available from the writers with the permission of the clinics and upon fair requests. It has been stated in our contract between the clinic and us that they never send us details about the participants because our data are part of a great database. Even they have their own competent statistics expert who analyzes our findings, the results were written based on his report.

\section{Funding}

Not applicable.

\section{Acknowledgements}

Not applicable.

\section{Competing interests}

The authors declare that there is no competing interests.

\section{Authors' contributions}

The project was designed by AM and AD; NB collected the samples wrote the draft paper; AD and AM reviewed and edited the paper. All authors read and approved the final manuscript.

\section{Authors' information}

Ariyo Movahedi, Email:amm35@mail.aub.edu

Abolghassem Djazayery'Email: djazayery@yahoo.com 


\section{References:}

1. Gerdle B, Ghafouri B, Ernberg M, Larsson B. Chronic musculoskeletal pain: Review of mechanisms and biochemical biomarkers as assessed by the microdialysis technique [Internet]. Vol. 7, Journal of Pain Research. Dove Medical Press Ltd.; 2014 [cited 2020 Oct 4]. p. 313-26. Available from: /pmc/articles/PMC4062547/?report=abstract

2. Cimmino MA, Ferrone C, Cutolo M. Epidemiology of chronic musculoskeletal pain. Vol. 25, Best Practice and Research: Clinical Rheumatology. Bailliere Tindall Ltd; 2011. p. 173-83.

3. Malik KM, Beckerly R, Imani F. Musculoskeletal disorders a universal source of pain and disability misunderstood and mismanaged: A critical analysis based on the U.S. model of care [Internet]. Vol. 8, Anesthesiology and Pain Medicine. Kowsar Medical Publishing Company; 2018 [cited 2020 Oct 8]. Available from: /pmc/articles/PMC6348332/?report=abstract

4. Mills SEE, Nicolson KP, Smith BH. Chronic pain: a review of its epidemiology and associated factors in population-based studies. Br J Anaesth [Internet]. 2019 Aug 1 [cited 2020 Dec 10];123(2):e273-83. Available from: /pmc/articles/PMC6676152/?report=abstract

5. Shane Anderson A, Loeser RF. Why is osteoarthritis an age-related disease? Vol. 24, Best Practice and Research: Clinical Rheumatology. 2010. p. 15-26.

6. Atella V, Piano Mortari A, Kopinska J, Belotti F, Lapi F, Cricelli C, et al. Trends in age-related disease burden and healthcare utilization. Aging Cell. 2019 Feb 1;18(1).

7. Babatunde OO, Jordan JL, Van Der Windt DA, Hill JC, Foster NE, Protheroe J. Effective treatment options for musculoskeletal pain in primary care: A systematic overview of current evidence. Vol. 12, PLoS ONE. Public Library of Science; 2017.

8. Chronic Pain - StatPearls - NCBI Bookshelf [Internet]. [cited 2020 Oct 8]. Available from: https://www.ncbi.nlm.nih.gov/books/NBK553030/

9. Elma Ö, Yilmaz ST, Deliens T, Coppieters I, Clarys P, Nijs J, et al. Do Nutritional Factors Interact with Chronic Musculoskeletal Pain? A Systematic Review. J Clin Med [Internet]. 2020 Mar 5 [cited 2020 Nov 21];9(3):702. Available from: /pmc/articles/PMC7141322/?report=abstract

10. Loeser RF. The Role of Aging in the Development of Osteoarthritis [Internet]. Vol. 128, Transactions of the American Clinical and Climatological Association. 2017 [cited 2019 Sep 29]. p. 44-54. Available from: http://www.ncbi.nlm.nih.gov/pubmed/28790486

11. Sophia Fox AJ, Bedi A, Rodeo SA. The basic science of articular cartilage: structure, composition, and function. Sports Health [Internet]. 2009 Nov;1(6):461-8. Available from: http://www.ncbi.nlm.nih.gov/pubmed/23015907

12. Bonjour J-P. Protein intake and bone health. Int J Vitam Nutr Res [Internet]. 2011 Mar;81(2-3):134-42. Available from: http://www.ncbi.nlm.nih.gov/pubmed/22139564

13. Nimni ME, Han B, Cordoba F. Are we getting enough sulfur in our diet? Vol. 4, Nutrition and Metabolism. 2007.

14. Zhu X, Sang L, Wu D, Rong J, Jiang L. Effectiveness and safety of glucosamine and chondroitin for the treatment of osteoarthritis: a meta-analysis of randomized controlled trials. J Orthop Surg Res [Internet]. 2018 Dec 6;13(1):170. Available from: https://josr-online.biomedcentral.com/articles/10.1186/s13018-0180871-5

15. Butawan M, Benjamin R, Bloomer R. Methylsulfonylmethane: Applications and Safety of a Novel Dietary Supplement. Nutrients [Internet]. 2017 Mar 16;9(3):290. Available from: http://www.mdpi.com/2072$6643 / 9 / 3 / 290$ 
16. Bin P, Huang R, Zhou X. Oxidation Resistance of the Sulfur Amino Acids: Methionine and Cysteine. Zhai L, editor. Biomed Res Int [Internet]. 2017;2017:9584932. Available from: https://doi.org/10.1155/2017/9584932

17. Górska-Warsewicz H, Laskowski W, Kulykovets O, Kudlińska-Chylak A, Czeczotko M, Rejman K. Food products as sources of protein and amino acids-The case of Poland. Nutrients [Internet]. 2018 Dec 13 [cited 2020 Oct 7];10(12). Available from: /pmc/articles/PMC6315330/?report=abstract

18. Ying J, Zhang M, Qiu X, Lu Y. The potential of herb medicines in the treatment of esophageal cancer. Vol. 103, Biomedicine and Pharmacotherapy. Elsevier Masson SAS; 2018. p. 381-90.

19. Khosravi M, Sadighi S, Moradi S, Zendehdel K. Persian-McGill pain questionnaire; translation, adaptation and reliability in cancer patients: A brief report. Tehran Univ Med J [Internet]. 2013 [cited 2019 Sep 26];71(1):53-8. Available from: http://tumj.tums.ac.ir

20. Roberts S, Colombier P, Sowman A, Mennan C, Rölfing JHD, Guicheux J, et al. Ageing in the musculoskeletal system. Acta Orthop [Internet]. 2016 Dec;87(sup363):15-25. Available from: http://www.ncbi.nlm.nih.gov/pubmed/27748151

21. Palmer KT, Goodson N. Ageing, musculoskeletal health and work. Best Pract Res Clin Rheumatol [Internet]. 2015 Jun;29(3):391-404. Available from: http://www.ncbi.nlm.nih.gov/pubmed/26612237

22. Lautenbacher S, Peters JH, Heesen M, Scheel J, Kunz M. Age changes in pain perception: A systematicreview and meta-analysis of age effects on pain and tolerance thresholds. Neurosci Biobehav Rev. 2017 Apr;75(4):104-13.

23. Minetto MA, Giannini A, McConnell R, Busso C, Torre G, Massazza G. Common Musculoskeletal Disorders in the Elderly: The Star Triad. J Clin Med [Internet]. 2020 Apr 23;9(4). Available from: http://www.ncbi.nlm.nih.gov/pubmed/32340331

24. Chen G, Zhang Y-Q, Qadri YJ, Serhan CN, Ji R-R. Microglia in Pain: Detrimental and Protective Roles in Pathogenesis and Resolution of Pain. Neuron [Internet]. 2018 Dec;100(6):1292-311. Available from: https://linkinghub.elsevier.com/retrieve/pii/S0896627318310006

25. Aloisi AM. Why We Still Need To Speak About Sex Differences and Sex Hormones in Pain. Pain Ther [Internet]. 2017 Dec [cited 2020 Nov 17];6(2):111-4. Available from: /pmc/articles/PMC5693815/?report=abstract

26. Wang Y-XJ, Griffith JF, Zeng X-J, Deng M, Kwok AWL, Leung JCS, et al. Prevalence and sex difference of lumbar disc space narrowing in elderly chinese men and women: osteoporotic fractures in men (Hong Kong) and osteoporotic fractures in women (Hong Kong) studies. Arthritis Rheum [Internet]. 2013 Apr;65(4):100410. Available from: http://www.ncbi.nlm.nih.gov/pubmed/23335175

27. Gioia C, Lucchino B, Tarsitano MG, Iannuccelli C, Di Franco M. Dietary habits and nutrition in rheumatoid arthritis: Can diet influence disease development and clinical manifestations? [Internet]. Vol. 12, Nutrients. MDPI AG; 2020 [cited 2020 Nov 28]. Available from: /pmc/articles/PMC7284442/?report=abstract

28. Bado A, Rozé C, Lewin MJM, Dubrasquet M. Endogenous opioid peptides in the control of food intake in cats. Peptides [Internet]. 1989 Sep;10(5):967-71. Available from: http://www.ncbi.nlm.nih.gov/pubmed/2558367

29. Elshorbagy AK, Church C, Valdivia-Garcia M, Smith AD, Refsum H, Cox R. Dietary cystine level affects metabolic rate and glycaemic control in adult mice. J Nutr Biochem [Internet]. 2012 Apr [cited 2020 Dec 6];23(4):332-40. Available from: /pmc/articles/PMC3315011/?report=abstract

30. Nimni ME, Han B, Cordoba F. Are we getting enough sulfur in our diet? [Internet]. Vol. 4, Nutrition and Metabolism. BioMed Central; 2007 [cited 2020 Dec 6]. p. 24. Available from: /pmc/articles/PMC2198910/?report=abstract 
31. Hruby A, Jacques PF. Dietary Protein and Changes in Biomarkers of Inflammation and Oxidative Stress in the Framingham Heart Study Offspring Cohort. Curr Dev Nutr [Internet]. 2019 May 1;3(5). Available from: https://academic.oup.com/cdn/article/doi/10.1093/cdn/nzz019/5421623

32. Delimaris I. Adverse Effects Associated with Protein Intake above the Recommended Dietary Allowance for Adults. ISRN Nutr [Internet]. 2013 [cited 2020 Nov 28];2013:1-6. Available from: /pmc/articles/PMC4045293/?report=abstract

33. Freyberg RH, Block WD, Fromer MF. A STUDY OF SULFUR METABOLISM AND THE EFFECT OF SULFUR ADMINISTRATION IN CHRONIC ARTHRITIS 1. J Clin Invest [Internet]. 1940 Mar 1 [cited 2020 Nov 27];19(2):423-35. Available from: https://www.ncbi.nlm.nih.gov/pmc/articles/PMC434976/

34. Benito-Garcia E, Feskanich D, Hu FB, Mandl LA, Karlson EW. Protein, iron, and meat consumption and risk for rheumatoid arthritis: A prospective cohort study. Arthritis Res Ther [Internet]. 2007 [cited 2020 Nov 28];9(1). Available from: https://pubmed.ncbi.nlm.nih.gov/17288585/

35. Hackney KJ, English KL. Protein and essential amino acids to protect musculoskeletal health during spaceflight: Evidence of a paradox? [Internet]. Vol. 4, Life. MDPI AG; 2014 [cited 2020 Nov 21]. p. 295-317. Available from: /pmc/articles/PMC4206848/?report=abstract

36. Ochi E, Tsuchiya Y. Eicosahexanoic acid (EPA) and docosahexanoic acid (DHA) in muscle damage and function [Internet]. Vol. 10, Nutrients. MDPI AG; 2018 [cited 2020 Dec 6]. Available from: /pmc/articles/PMC5986432/?report=abstract

37. Silva R V, Oliveira JT, Santos BLR, Dias FC, Martinez AMB, Lima CKF, et al. Long-Chain Omega-3 Fatty Acids Supplementation Accelerates Nerve Regeneration and Prevents Neuropathic Pain Behavior in Mice. Front Pharmacol. 2017;8:723.

38. Bin P, Huang R, Zhou X. Oxidation resistance of the sulfur amino acids: Methionine and cysteine. Vol. 2017, BioMed Research International. Hindawi Limited; 2017.

39. Demirkol O, Ercal N. Glutathione. In: Handbook of Analysis of Active Compounds in Functional Foods [Internet]. CRC Press; 2012 [cited 2020 Nov 27]. p. 68-85. Available from: https://www.ncbi.nlm.nih.gov/pmc/articles/PMC4684116/

40. Pizzorno J. Glutathione! Integr Med (Encinitas) [Internet]. 2014 Feb;13(1):8-12. Available from: http://www.ncbi.nlm.nih.gov/pubmed/26770075

41. Stone LS, Molliver DC. In search of analgesia: emerging roles of GPCRs in pain. Mol Interv [Internet]. 2009 Oct;9(5):234-51. Available from: http://www.ncbi.nlm.nih.gov/pubmed/19828831

42. Ghodoosi N, Mirzababaei A, Rashidbeygi E, Badrooj N, Sajjadi SF, Setayesh L, et al. Associations of dietary inflammatory index, serum levels of MCP-1 and body composition in Iranian overweight and obese women: a cross-sectional study. BMC Res Notes [Internet]. 2020 Dec 1 [cited 2020 Dec 27];13(1). Available from: /pmc/articles/PMC7684955/?report=abstract

43. Nalamachu S. An overview of pain management: the clinical efficacy and value of treatment. Am J Manag Care [Internet]. 2013 Nov;19(14 Suppl):s261-6. Available from: http://www.ncbi.nlm.nih.gov/pubmed/24494608

44. Pujos-Guillot E, Pickering G, Lyan B, Ducheix G, Brandolini-Bunlon M, Glomot F, et al. Therapeutic paracetamol treatment in older persons induces dietary and metabolic modifications related to sulfur amino acids. Age (Omaha) [Internet]. 2012 Feb [cited 2020 Nov 26];34(1):181-93. Available from: /pmc/articles/PMC3260351/?report=abstract

45. Veeranki S, Tyagi SC. Defective homocysteine metabolism: Potential implications for skeletal muscle malfunction [Internet]. Vol. 14, International Journal of Molecular Sciences. Multidisciplina- 
ry Digital Publishing Institute (MDPI); 2013 [cited 2020 Nov 27]. p. 15074-91. Available from: /pmc/articles/PMC3742288/?report=abstract

46. Goldstein DS. Catecholamines 101 [Internet]. Vol. 20, Clinical Autonomic Research. Dr. Dietrich Steinkopff Verlag GmbH and Co. KG; 2010 [cited 2020 Dec 6]. p. 331-52. Available from: /pmc/articles/PMC3046107/?report=abstract

47. Hannibal KE, Bishop MD. Chronic stress, cortisol dysfunction, and pain: A psychoneuroendocrine rationale for stress management in pain rehabilitation. Phys Ther [Internet]. 2014 Dec 1 [cited 2020 Dec 6];94(12):1816-25. Available from: /pmc/articles/PMC4263906/?report=abstract 